02

\title{
Электронно-дырочные димеры в „родительской“ фазе квази-2D-купратов
}

\author{
() А.С. Москвин, Ю.Д. Панов \\ Уральский федеральный университет, \\ Екатеринбург, Россия \\ E-mail: alexander.moskvin@urfu.ru
}

Поступила в Редакцию 15 апреля 2019 г.

В окончательной редакции 22 апреля 2019 г.

Принята к публикации 24 апреля 2019 г.

\begin{abstract}
Показано, что наряду с высокой ионной поляризуемостью и близостью к „поляризационной катастрофе“ важнейшей особенностью родительских купратов типа $\mathrm{La}_{2} \mathrm{CuO}_{4}$, предопределяющей их необычное поведение при неизовалентном замещении, является неустойчивость относительно переноса заряда с формированием системы метастабильных дипольно-активных „мотт-хаббардовских“ экситонов - электронно-дырочных (ЕН) димеров. Неизовалентное замещение смещает фазовое равновесие в сторону конденсации ЕН-димеров и формирования неоднородной ЕН-жидкости, в простейшей модели эквивалентной системе композитных бозонов. Для эффективного описания электронного состояния допированных купратов предлагается использовать $S=1$ псевдоспиновый формализм, который позволяет рассмотреть принципиально новые зарядовые состояния типа RVB-фазы Андерсона. Рекомбинация ЕН-димеров при критически малом значении энергии локальных и нелокальных корреляций приводит к переходу системы в ферми-жидкостное состояние.
\end{abstract}

Ключевые слова: купраты, электронная структура, электронно-дырочные димеры.

DOI: $10.21883 /$ FTT.2019.09.48097.27N

\section{1. Введение}

Природа высокотемпературной сверхпроводимости (BТСП) и других аномальных свойств квази-2D-купратов типа $\mathrm{La}_{2-x} \mathrm{Sr}_{x} \mathrm{CuO}_{4}$, так или иначе, связана со специфическими свойствами „родительской“ фазы. Замечательной особенностью родительских купратов типа $\mathrm{La}_{2} \mathrm{CuO}_{4}, \mathrm{YBa}_{2} \mathrm{Cu}_{3} \mathrm{O}_{6}$ является высокая ионная поляризуемость, а также аномально большие значения диэлектрических констант $[1,2]$. В простейшей изотропной модели диэлектрическая проницаемость ионного кристалла содержит фактор „усиления“

$$
\left[1-\frac{4 \pi}{3} \sum_{i} n_{i} \alpha_{i}\right]^{-1},
$$

где $n_{i}-$ плотность, $\alpha_{i}-$ поляризуемость ионов. Поляризационный фактор $S=\frac{4 \pi}{3} \sum_{i} n_{i} \alpha_{i}$ (refractivity sum) для родительских купратов близок к единице [2], что означает их близость к „поляризационной катастрофе“. Такие системы неустойчивы относительно перехода в новое состояние с конденсацией локальных или коллективных низкоэнергетических возбуждений типа однородного или „зарождающегося“ (incipient) ферроэлектрика, перехода изолятор - металл, различных вариантов зарядового упорядочения. Конкретный вариант зависит от характера низкоэнергетических возбуждений, конкуренции различных степеней свободы и соответствующих параметров порядка. Основным кандидатом в родительских купратах являются зарядово-нейтральные, но дипольно-активные „мотт-хаббардовские“ экситоны с переносом заряда. Минимальная энергия, необходимая для рождения такой электронно-дырочной (EH) пары путем прямого франк-кондоновского (FC) оптического перехода с переносом заряда в родительских купратах, то есть оптическая щель, составляет $E_{\mathrm{gap}}^{\mathrm{opt}} \approx 1.5-2.0 \mathrm{eV}$. Фактически это энергия образования нестабильного экситона с переносом заряда, или ЕН-димера, как своеобразного кванта реакции диспропорционирования. Эффекты электрон-решеточной релаксации приводят либо к его распаду с ЕН-рекомбинацией, либо к образованию метастабильного ЕН-димера, устойчивость которого поддерживается локальной деформацией решетки и электронной поляризацией окружения. Энергия метастабильного ЕН-димера определяет „адиабатическую“ или „термическую“ щель с переносом заряда, которая может существенно отличаться от оптической щели $[3,4]$. К сожалению, экспериментальная информация об энергии релаксации в родительских купратах крайне скудна. Так, высокотемпературные холловские измерения позволили оценить энергию образования пары несвязанных электронных и дырочных носителей в родительском купрате $\mathrm{La}_{2} \mathrm{CuO}_{4}$ [5]: $U_{\text {th }}=0.89 \mathrm{eV}$. Измерения химических потенциалов дырок и электронов в $\mathrm{Y}_{0.38} \mathrm{La}_{0.62} \mathrm{Ba}_{1.74} \mathrm{La}_{0.26} \mathrm{Cu}_{3} \mathrm{O}_{y} \quad$ (YLBLCO) [6] дают для этой энергии величину порядка $0.8 \mathrm{eV}$. Это означает, что минимальная энергия $E_{\text {gap }}^{\text {th }}$ образования связанных в ЕН-димере электронных и дырочных центров должна быть существенно меньше $0.9 \mathrm{eV}$, что указывает на неустойчивость родительских купратов относительно переноса заряда с образованием устойчивых ЕН-димеров. Эта энергия может быть иден- 
тифицирована как низкоэнергетический край слабой „не-франк-кондоновской“ (NFC) полосы, отчетливо видимой в среднем ИК-диапазоне $0.4-1.0 \mathrm{eV}$ (MIRполоса) во всех родительских купратах и в определенном смысле являющейся их „визитной карточкой“ [3]. С учетом $E_{\text {gap }}^{\text {th }} \approx 0.4 \mathrm{eV}$ мы получаем для энергии связи электронных и дырочных центров в $\mathrm{La}_{2} \mathrm{CuO}_{4}$ величину $V_{\mathrm{EH}} \approx 0.5 \mathrm{eV}$. Нужно отметить, что на краю достаточно широкой MIR-полосы в купратах обнаруживается узкий пик, связанный с двухмагнонным (2M) поглощением. Кстати, подобный двухмагнонный пик является единственной в MIR-диапазоне спектральной особенностью изоструктурного купратам антиферромагнетика $\mathrm{La}_{2} \mathrm{NiO}_{4}$ [7], что подчеркивает уникальность купратов как систем, неустойчивых относительно переноса заряда и формирования устойчивых ЕН-димеров - низкоэнергетических зарядовых (но нейтральных!) возбуждений. Эта неустойчивость является результатом сильного электронно-колебательного взаимодействия, которое является важным „участником“ формирования необычных свойств купратов. Близость энергий спиновых и зарядовых возбуждений в родительских купратах свидетельствует о конкуренции соответствующих степеней свободы в „борьбе“ за основное состояние.

В данной работе мы рассматриваем свойства ЕН-димеров, их роль в формировании необычного нормального и сверхпроводящего состояния купратов, а также „нечастичный“ (unparticle) $S=1$ псевдоспиновый формализм описания зарядовых состояний.

\section{2. ЕН-димеры в родительских купратах}

Устойчивые ЕН-димеры, или „мотт-хаббардовсие“ $d-d$-экситоны с переносом заряда, в родительских купратах представляют собой связанные электронный $\left[\mathrm{CuO}_{4}\right]^{7-}$ и дырочный $\left[\mathrm{CuO}_{4}\right]^{5-}$ центры, соответствующие кластеру $\mathrm{CuO}_{4}$ с полностью заполненными $\mathrm{Cu} 3 d$ - и О $2 p$-оболочками, или вакуумному состоянию для дырок $|0\rangle$, и двухдырочной конфигурации $|2\rangle$ кластера $\mathrm{CuO}_{4}$ с основным жанг-райсовским (Zhang-Rice) синглетом. Дублет $|02\rangle,|20\rangle$ расщепляется благодаря резонансной реакции двухчастичного переноса $|02\rangle \leftrightarrow|20\rangle$. Новые суперпозиции

$$
| \pm\rangle=\frac{1}{\sqrt{2}}=(|02\rangle \pm|20\rangle)
$$

с энергией $E_{ \pm}=U_{\mathrm{th}}-V_{\mathrm{EH}} \pm\left|t_{\mathrm{B}}\right|$ образуют димеры $S$ $(|+\rangle)$ и Р- $(|-\rangle)$ типа, где $U_{\text {th }}-$ энергия образования пары несвязанных электронных и дырочных носителей, $V_{\text {Ен }}$ - энергия связи электронных и дырочных центров в родительском купрате. Величина эффективного интеграла двухчастичного переноса $t_{\mathrm{B}}=\langle 20|H| 02\rangle$, определяющего $\mathrm{S}$-P-расщепление, играет принципиальную роль в „судьбе“ родительских купратов. Дело в том, что этот интеграл фактически является интегралом переноса локального композитного бозона, образуемого парой

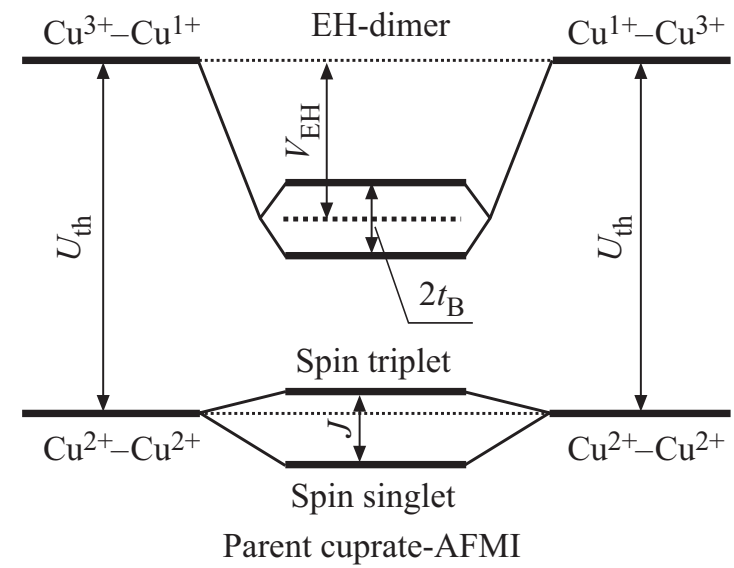

Рис. 1. Спектр ЕН-димера в родительском купрате с учетом электростатического ЕН-взаимодействия и двухчастичного переноса.

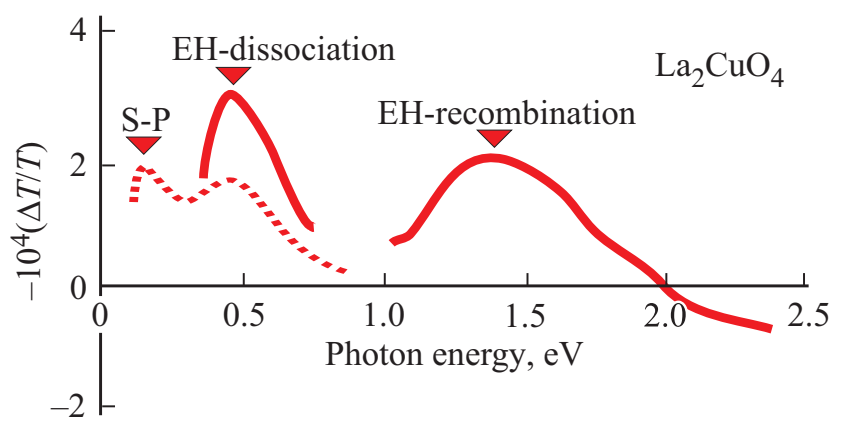

Рис. 2. Сплошные кривые - спектр фотоиндуцированного поглощения $\mathrm{La}_{2} \mathrm{CuO}_{4}$ при $15 \mathrm{~K}$ и энергии фотонов накачки $2.54 \mathrm{eV}$ [8]. Пунктирная кривая - спектр фотоиндуцированного поглощения $\mathrm{La}_{2} \mathrm{CuO}_{4}$ при $4.2 \mathrm{~K}$ и энергии фотонов накачки $2.7 \mathrm{eV}$ [9].

частиц, электронов или дырок, локализованных на одном кластере $\mathrm{CuO}_{4}$ и формально различающих электронный и дырочный центры.

Независимые экспериментальные данные для различных купратов указывают на величину $t_{\mathrm{B}} \approx 0.1 \mathrm{eV}[3,4]$, близкую, как и следовало ожидать, к величине обменного интеграла в родительском купрате. Интересно, что формально ЕН-димеры можно рассматривать как центры с отрицательной энергией корреляции $U$ (negative- $U$ centers). На рис. 1 для иллюстрации представлена энергетическая структура изолированной пары ближайших центров $\mathrm{CuO}_{4}$ с учетом переноса заряда, связи электронного и дырочного центров, а также изотропного обмена центров $\left[\mathrm{CuO}_{4}\right]^{6-}$ (номинально $\mathrm{Cu}^{2+}$-центров) со спином $1 / 2$, как в родительском купрате, так и, предположительно, при относительно небольшом электронном или дырочном допировании.

ЕН-димеры проявляются в различных оптических свойствах родительских купратов, в частности, в спектрах фотоиндуцированного поглощения. 
На рис. 2 представлены спектры фотоиндуцированного поглощения $\mathrm{La}_{2} \mathrm{CuO}_{4}$ при $15 \mathrm{~K}$ и энергии фотонов накачки $2.54 \mathrm{eV}[8]$, а также при $4.2 \mathrm{~K}$ и энергии фотонов накачки $2.7 \mathrm{eV}$ [9]. Пик при $0.5 \mathrm{eV}$ может быть естественным образом связан с фотодиссоциацией ЕН-димеров, в то время как высокоэнергетический пик при $1.4 \mathrm{eV}$ может быть связан с фоторекомбинацией ЕН-димеров, то есть обратным переходом с переносом заряда и аннигиляцией ЕН-пары. Пик при $0.2 \mathrm{eV}$ приписывается S-P-переходу в фотоиндуцированных ЕН-димерах. Подобные, хотя и относительно слабые пики, наблюдаются практически во всех родительских купратах [3,4], свидетельствуя о существовании в них небольшой концентрации устойчивых ЕН-димеров. Огромная величина электрического дипольного момента $\mathrm{S}-\mathrm{P}$-перехода: $d_{S P}=|\langle S|d| P\rangle| \approx 2 e R_{\mathrm{CuCu}} \approx 8 e\left(R_{\mathrm{CuCu}}\right.$ в ангстремах) указывает на важную роль $\mathrm{S}-$ Р-дублета в нелинейной оптике родительских купратов, в частности, в электроотражении, двухфотонном поглощении и генерации третьей гармоники. Принципиально важное значение для купратов приобретает аномально высокая электрическая поляризуемость ЕН-димеров, которые можно рассматривать как зародыши новой высокополяризуемой фазы, переход в которую „поляризационнонеустойчивой“ ${ }^{\prime \prime}$ родительской фазы может быть либо спонтанным, либо индуцированным, например, неизовалентным замещением.

\section{3. ЕН-димеры и особенности допированных купратов}

При конечных температурах родительские купраты представляют собой системы с малой концентрацией метастабильных ЕН-димеров, гигантская электрическая поляризуемость которых, действительно приводит к аномальному поведению купратов при неизовалентном замещении в системах типа $\mathrm{La}_{2-x} \mathrm{Sr}_{x} \mathrm{CuO}_{4}$, $\mathrm{Nd}_{2-x} \mathrm{Ce}_{x} \mathrm{CuO}_{4}, \mathrm{YBa}_{2} \mathrm{Cu}_{3} \mathrm{O}_{6+x}$ и $\mathrm{La}_{2} \mathrm{CuO}_{4+\delta}$, сопровождаемом появлением неоднородного электрического потенциала и электронным или дырочным допированием. Рост концентрации центров примесного электрического потенциала сопровождается конденсацией и ростом концентрации ЕН-димеров, обеспечивающих эффективную экранировку примесного потенциала, с одновременным ростом энергии электрон-решеточной релаксации и резким понижением как энергии переноса заряда, так и

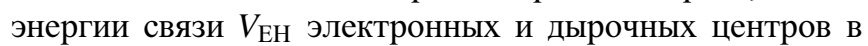
ЕН-димерах. Так, по данным высокотемпературных холловских измерений [5] энергия образования пары несвязанных электронных и дырочных носителей $U_{\text {th }}$ резко падает с 0.89 до $0.53 \mathrm{eV}$ при замещении всего лишь $1 \%$ трехвалентных ионов $\mathrm{La}^{3+}$ двухвалентными ионами $\mathrm{Sr}^{2+}$ в родительском купрате $\mathrm{La}_{2} \mathrm{CuO}_{4}$ и продолжает резко падать при дальнейшем росте допирования. На рис. 3 представлена зависимость энергии образования несвязанных электронов и дырок от уровня допирования в

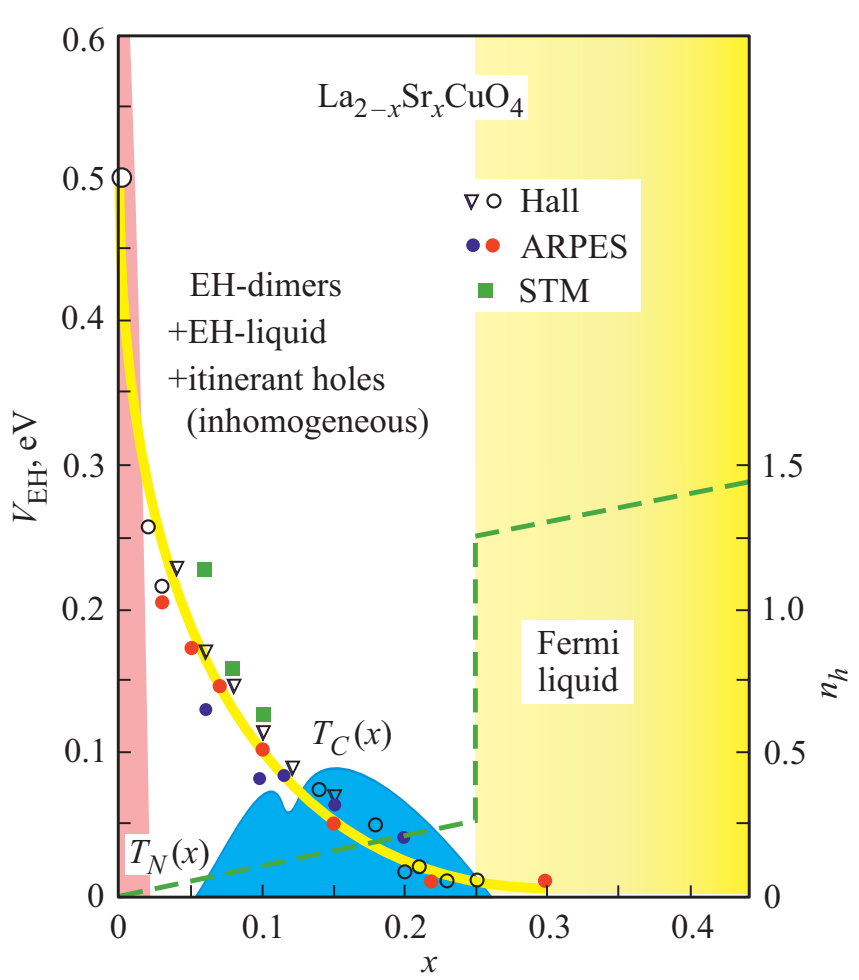

Рис. 3. Модельная „фазовая диаграмма“ купрата $\mathrm{La}_{2-x} \mathrm{Sr}_{x} \mathrm{CuO}_{4}$. Зависимость энергии ЕН-связи в димере $V_{\mathrm{EH}}$ от уровня допирования показана по данным [11] и [3] $\left(V_{\text {ЕH }}\right.$ при $\left.x=0\right)$. Пунктирной линией показана зависимость концентрации носителей от уровня допирования со скачком в точке ЕН-рекомбинации. Для сравнения приведены соответствующие зависимости темепературы Нееля и температуры сверхпроводящего перехода.

$\mathrm{La}_{2-x} \mathrm{Sr}_{x} \mathrm{CuO}_{4}$ по данным работ $[3,5,10,11]$. Горьков и Тейтельбаум [11] связывают энергию $V_{\mathrm{EH}} \mathrm{c}$ характерной температурой $T^{*}$, определяющей не совсем четко определенные границы „псевдощелевой“ фазы [12], однако на наш взгляд существует, как минимум два „кандидата“ на характерную энергию псевдощели $-E_{\text {gap }}^{\text {th }}$ и $V_{\text {ЕH }}$, что вполне согласуется с экспериментально наблюдаемым „дублетом“ температур псевдощели $T^{*}$ [12]. Очевидно, что эффект экранировки локальных и нелокальных корреляций - потенциальных электростатических энергий $U_{\text {th }}$ и $V_{\text {EH, }}$, не должен приводить к существенному влиянию на параметры кинетической энергии типа интегралов двухчастичного переноса $t_{\mathrm{B}}$. Рост концентрации ЕН-димеров, сопровождаемый резким понижением энергии связи электронных и дырочных центров, приводит к их разрушению с образованием своеобразной сильнокоррелированной электронно-дырочной ЕН-жидкости. EH-жидкость в традиционных полупроводниках типа $\mathrm{Ge}$, представляет собой двухкомпонентную ферми-жидкость, тогда как ЕН-жидкость в купратах представляет собой систему сильнокоррелированных электронных и дырочных центров, в простейшей модели эквивалентную бозе-жидкости [3] с возможностью формирова- 
ния сверхпроводящего бозе-эйнштейновского конденсата при некоторой критической величине допирования. Реальная ситуация в допированных купратах с экранированными параметрами $U_{\text {th }}$ и $V_{\mathrm{EH}}$ предполагает рассмотрение „бозон-фермионной“ системы $\mathrm{CuO}_{4}$-центров в $\mathrm{CuO}_{2}$-плоскостях, которые могут находиться в трех близких по энергии различных валентных зарядовых состояниях: $\left[\mathrm{CuO}_{4}\right]^{7-, 6-, 5-}$ (номинально $\left.\mathrm{Cu}^{1+, 2+, 3+}\right)$. Этот зарядовый триплет можно формально связать с тремя состояниями псевдоспина $S=1(M=-1,0,+1$ соответственно) и использовать известные методы описания спиновых систем $[3,13]$.

\section{4. Эфффективный $S=1$ псевдоспиновый гамильтониан купрата}

Псевдоспиновый формализм для системы многоэлектронных центров со смешанной валентностью представляет собой пример „нечастичного“ (unparticle) описания, наиболее эффективного в случае сильнокоррелированных систем. Спиновая алгебра $S=1\left(M_{S}=0, \pm 1\right)$ включает восемь независимых операторов (три дипольных и пять квадрупольных)

$$
S_{z}, S_{ \pm}=\mp \frac{1}{\sqrt{2}}\left(S_{x} \pm i S_{y}\right) ; S_{z}^{2} ; T_{ \pm}=\left\{S_{z}, S_{ \pm}\right\} ; S_{ \pm}^{2} .
$$

Операторы повышения/понижения $S_{ \pm}$и $T_{ \pm}$меняют проекцию псевдоспина на \pm 1

$$
\begin{aligned}
& \left\langle 0\left|S_{ \pm}\right| \mp 1\right\rangle=\left\langle \pm 1\left|S_{ \pm}\right| 0\right\rangle=\mp 1 \\
& \left\langle 0\left|T_{ \pm}\right| \mp 1\right\rangle=-\left\langle \pm 1\left|T_{ \pm}\right| 0\right\rangle=+1,
\end{aligned}
$$

то есть фактически являются операторами рождения/уничтожения электрона/дырки в многочастичном атомном состоянии. Удобнее использовать комбинированные операторы

$$
P_{ \pm}=\frac{1}{2}\left(S_{ \pm}+T_{ \pm}\right) ; \quad N_{ \pm}=\frac{1}{2}\left(S_{ \pm}-T_{ \pm}\right),
$$

которые описывают переходы $\mathrm{Cu}^{2+} \leftrightarrow \mathrm{Cu}^{3+}$ и $\mathrm{Cu}^{2+} \leftrightarrow \mathrm{Cu}^{1+}$ соответственно. Операторы повышения/понижения $S_{ \pm}^{2}$ меняют проекцию псевдоспина на \pm 2 и описывают переходы $\mathrm{Cu}^{1+} \leftrightarrow \mathrm{Cu}^{3+}$. Узельный недиагональный параметр порядка $\left\langle S_{ \pm}^{2}\right\rangle$ отличен от нуля только если на узле имеется квантовая суперпозиция $\mathrm{Cu}^{1+} \leftrightarrow \mathrm{Cu}^{3+}$. Оператор $S_{+}^{2}\left(S_{-}^{2}\right)$ создает на узле дырочную (электронную) пару, или эффективный локальный композитный бозон, с кинематическим ограничением $\left(S_{ \pm}^{2}\right)^{2}=0$, что подчеркивает его природу как „жесткого“ (hard-core) бозона. Среднее

$$
\Psi=\left\langle S_{ \pm}^{2}\right\rangle=\frac{1}{2}\left\langle S_{x}^{2}-S_{y}^{2}\right\rangle \pm i\left\langle\left\{S_{x}, S_{y}\right\}\right\rangle
$$

служит $d$-симметричным параметром локального сверхпроводящего порядка.
Эффективный $S=1$ псевдоспиновый гамильтониан системы зарядовых триплетов в купратах, который коммутирует с $z$-компонентой полного псевдоспина $\sum_{i} S_{i z}$ и, таким образом, сохраняет полный заряд системы, представим как сумму потенциальной и кинетической энергий:

$$
\begin{gathered}
H=H_{\mathrm{pot}}+H_{\mathrm{kin}}^{(1)}+H_{\mathrm{kin}}^{(2)}, \\
H_{\mathrm{pot}}=\sum_{i}\left(\Delta S_{i z}^{2}-\mu S_{i z}\right)+\frac{1}{2} \sum_{i j} V_{i j} S_{i z} S_{j z}, \\
H_{\mathrm{kin}}^{(1)}=\frac{1}{2} \sum_{i j}\left[t^{p} P_{i+} P_{j-}+t^{n} N_{i+} N_{j-}\right. \\
\left.+\frac{1}{2} t^{p n}\left(P_{i+} N_{j-}+N_{i+} P_{j-}\right)+\text { h.c. }\right], \\
H_{\mathrm{kin}}^{(2)}=-\frac{1}{2} \sum_{i j} t_{i j}^{b}\left(S_{i+}^{2} S_{j-}^{2}+S_{i-}^{2} S_{j+}^{2}\right) .
\end{gathered}
$$

За исключением некоторых слагаемых, неинвариантных относительно обращения времени в (4), этот гамильтониан представляет один из наиболее общих анизотропных $S=1$ спин-гамильтонианов. Первое слагаемое в (3), или „одноионная анизотропия“, описывает корреляционные эффекты плотность-плотность на узлах $(2 \Delta=U)$. Второе слагаемое может быть связано с псевдомагнитным полем вдоль оси $O_{z}$, или с химическим потенциалом относительно добавления новых частиц. Последний член в (3) описывает межузельные взаимодействия (корреляции) типа плотность-плотность. Гамильтониан (4) ( $X Y$-анизотропия) описывает три типа „одночастичного“ коррелированного переноса, а гамильтониан (5) (биквадратичная двухцентровая анизотропия) - „Двухчастичный“ транспорт, или перенос композитных бозонов. Эффективный гамильтониан $\hat{H}$ описывает многообразие типов „диагонального“ и „недиагонального“ зарядового порядка от диэлектрической фазы родительского купрата (аналог квантового парамагнетика), волн зарядовой (псевдоспиновой) плотности, сверхпроводимости. Отметим, что псевдоспиновый формализм позволяет естественным образом обобщить метод RVB (resonating valence bonds) Андерсона [14] на описание специфического квантового зарядового упорядочения в купратах типа псевдоспиновой жидкости.

На рис. 3 представлена модельная „фазовая диаграмма“ купрата $\mathrm{La}_{2-x} \mathrm{Sr}_{x} \mathrm{CuO}_{4}$. Зависимость энергии ЕН-связи в димере $V_{\text {ЕН }}$ от уровня допирования приведена согласно работ данным [11] и [3] ( $V_{\mathrm{EH}}$ при $\left.x=0\right)$. Рекомбинация ЕН-димеров при критически малом значении энергии локальных и нелокальных корреляций приводит к переходу системы в ферми-жидкостное состояние при $x=x_{c} \approx 0.25$ с резким ростом концентрации дырочных носителей от $n_{h}=x_{c}$ до $n_{h}=1+x_{c}$. Зависимость $n_{h}(x)$ показана пунктирной линией. Для сравнения на рисунке приведены соответствующие зависимости температуры Нееля и температуры сверхпроводящего перехода. 


\section{5. Конкуренция зарядовой и спиновой степеней свободы в купратах}

Псевдоспиновое описание зарядовой степени свободы соответствует ,диэлектрическому“ сценарию физики купратов (см., например, [2]), в рамках которого мы пренебрегаем спиновой степенью свободы подобно приближению Хартри в отличии от приближения Хартри-Фока. Сверхобменное взаимодействие $\mathrm{Cu}^{2+}-\mathrm{Cu}^{2+}$ мы можем учесть, включив в $\hat{H}$ спингамильтониан Гейзенберга в виде

$$
H_{e x}=\sum_{\langle i j\rangle} \hat{J}_{i j}\left(s_{i} s_{j}\right),
$$

где $\hat{J}_{i j}$ фактически является псевдоспиновым оператором

$$
\hat{J}_{i j}=\hat{P}_{i} J_{i j} \hat{P}_{j},
$$

где $J_{i j}-$ обычный сверхобменный интеграл, $\hat{P}_{i}=\left(1-S_{i z}^{2}\right) \quad-\quad$ оператор проектирования на псевдоспиновое состояние $M=0$, то есть $\mathrm{Cu}^{2+}$.

Наряду с ,диэлектрическим“ остается популярным и „магнитный“ сценарий ВТСП купратов, предполагающий ведущую роль спиновой степени свободы (см., например, [15]). Так, в ряде купратов, например, в системе $\left(\mathrm{Ca}_{x} \mathrm{La}_{1-x}\right)\left(\mathrm{Ba}_{1.75-x} \mathrm{La}_{0.25+x}\right) \mathrm{Cu}_{3} \mathrm{O}_{y}$ установлена корреляция между величиной обменных интегралов и максимальной $T_{c}$ [16], что рассматривается важным аргументом в пользу „магнитного“ сценария. Абсолютно иная ситуация наблюдается для ряда изоструктурных купратов $\mathrm{RBa}_{2-x} \mathrm{Sr}_{x} \mathrm{Cu}_{3} \mathrm{O}_{y}(\mathrm{R}=\mathrm{La}, \mathrm{Nd}, \mathrm{Sm}, \mathrm{Gd}, \mathrm{Dy}, \mathrm{Yb})$, где обнаружена антикорреляция $T_{c \max }$ с величинами обменных интегралов [2], но корреляция между $T_{c} \max$ и величиной поляризационного фактора $S=\frac{4 \pi}{3} \sum_{i} n_{i} \alpha_{i}$, что прямо указывает на диэлектрический сценарий ВТСП. Однако, наличие корреляции между максимальным значением $T_{c}$ и величиной обменного интеграла, а тем более, величиной $T_{N}$, не является аргументом в пользу „магнитного“ сценария ВТСП. Дело в том, что в простейшей модели „замороженной“ решетки обменный интеграл $J$ фактически совпадает с интегралом переноса композитного бозона $t_{\mathrm{B}}$, так что в модели $\mathrm{EH-жидкости}$ с ростом $J$ естественно ожидать и роста $T_{c}$. Однако реальная ситуация выглядит сложнее. Температура сверхпроводящего перехода в модельной ЕН-жидкости определяется конкуренцией кинетической и потенциальной энергий, в простейшем приближении определяемых интегралом переноса композитного бозона $t_{\mathrm{B}}$ и параметрами $U_{\text {th }}$ и $V_{\text {ЕН }}$ соответственно. Таким образом, в купратах типа $\left(\mathrm{Ca}_{x} \mathrm{La}_{1-x}\right)\left(\mathrm{Ba}_{1.75-x} \mathrm{La}_{0.25+x}\right) \mathrm{Cu}_{3} \mathrm{O}_{y}$ корреляция $T_{c \max } \mathrm{c} J$ [16] просто отражает естественную корреляцию $T_{c \text { max }}$ с $t_{\text {B }}$ тогда как в купратах $\mathrm{RBa}_{2-x} \mathrm{Sr}_{x} \mathrm{Cu}_{3} \mathrm{O}_{y}$ преобладание кинетического вклада в формирование сверхпроводимости с ростом $T_{c \max }$ достигается за счет эффективного подавления потенциального вклада. Действительно, рост диэлектрической постоянной приводит к большей экранировке электростатики, понижению $U_{\text {th }}$

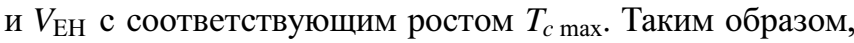
обе группы результатов могут быть объяснены в рамках „диэлектрического“ сценария. Фактически, спиновая степень свободы в модели ЕН-жидкости играет, скорее всего, „паразитную“ роль, приводя к редукции кинетической энергии композитных бозонов, а значит и $T_{c} \max$.

\section{6. Заключение}

Кроме высокой ионной поляризуемости и аномально больших значений диэлектрических констант, родительские купраты типа $\mathrm{La}_{2} \mathrm{CuO}_{4}$ характеризуются неустойчивостью относительно переноса заряда с формированием системы метастабильных дипольно-активных „моттхаббардовских“ экситонов - электронно-дырочных (EH) димеров. Неизовалентное замещение в таких купратах смещает фазовое равновесие в сторону конденсации ЕН-димеров и формирования неоднородной ЕН-жидкости, в простейшей модели эквивалентной системе композитных бозонов - пар электронов/дырок, различающих бесспиновые электронный $\left[\mathrm{CuO}_{4}\right]^{7-}$ и дырочный $\left[\mathrm{CuO}_{4}\right]^{5-}$ центры. Для эффективного описания системы трех многоэлектронных зарядовых центров $\left[\mathrm{CuO}_{4}\right]^{7-, 6-, 5-}$ в допированных купратах предлагается использовать „нечастичный“ $S=1$ псевдоспиновый формализм, который позволяет с единых позиций рассмотреть большое многообразие фазовых состояний, включая принципиально новые зарядовые состояния типа $R V B$-фазы Андерсона - квантовую псевдоспиновую ЕН-жидкость. Рекомбинация ЕН-димеров при критически малом значении энергии локальных и нелокальных корреляций приводит к переходу системы в фермижидкостное состояние. $\mathrm{B} \mathrm{La}_{2-x} \mathrm{Sr}_{x} \mathrm{CuO}_{4}$ такой переход реализуется при $x \approx 0.25$ и характеризуется резким увеличением концентрации дырочных носителей.

В рамках модели зарядовых триплетов купраты попадают в универсальный класс (псевдо)спиновых 2D-систем, для которых необходимым элементом термодинамического описания являются различные неоднородные структуры, домены и доменные стенки, топологические дефекты типа вихрей, скирмионов, характеризуемые пространственно-неоднородным распределением псевдоспиновой плотности [17]. В частности, они включают области филаментарной и локальной сверхпроводимости, существующие и при температурах, существенно превышающих $T_{c}$.

\section{Финансирование работы}

Работа выполнена при поддержке Программы 211 Правительства Российской Федерации, соглашение № 02.А03.21.0006, и проектов № 2277 и № 5719 Министерства Образования и Науки Российской Федерации. 


\section{Конфликт интересов}

Авторы заявляют, что у них нет конфликта интересов.

\section{Список литературы}

[1] D. Reagor, E. Ahrens, S.W. Cheong, A. Migliori, Z. Fisk. Phys. Rev. Lett. 62, 2048 (1989).

[2] B.P.P. Mallett, T. Wolf, E. Gilioli, F. Licci, G.V.M. Williams, A.B. Kaiser, N.W. Ashcroft, N. Suresh, J.L. Tallon. Phys. Rev. Lett. 111, 237001 (2013).

[3] A.S. Moskvin. Phys. Rev. B 84, 075116 (2011).

[4] А.С. Москвин. ФТТ 61, 809 (2019).

[5] S. Ono, S. Komiya, Y. Ando. Phys. Rev. B 75, 024515 (2007).

[6] M. Ikeda, M. Takizawa, T. Yoshida, A. Fujimori, K. Segawa, Y. Ando. Phys. Rev. B 82, 020503(R) (2010).

[7] M.A. Kastner, R.J. Birgeneau, G. Shirane, Y. Endoh. Rev. Mod. Phys. 70, 897 (1998); M. Grüninger, J. Münzel, A. Gaymann, A. Zibold, H.P. Geserich, T. Kopp. Europhys. Lett. 35, 55 (1996).

[8] J.M. Ginder, M.G. Roe, Y. Song, R.P. McCall, J.R. Gaines, E. Ehrenfreund, A.J. Epstein. Phys. Rev. B 37, 7506 (1988).

[9] Y.H. Kim, S.-W. Cheong, Z. Fisk. Phys. Rev. Lett. 67, 2227 (1991).

[10] Y. Ando, Y. Kurita, S. Komiya, S. Ono, K. Segawa. Phys. Rev. Lett. 92, 197001 (2004).

[11] L.P. Gorkov, G.B. Teitelbaum. Phys. Rev. Lett. 97, 247003 (2006); J. Phys.: Conf. Ser. 108, 12009 (2008).

[12] T. Honma, P.H. Hor. Phys. Rev. B 77, 184520 (2008).

[13] A.S. Moskvin. J. Phys.: Conf. Ser. 592, 012076 (2015); ЖЭТФ, 121, 549 (2015).

[14] P.W. Anderson. Science. 235, 1196 (1987).

[15] D.J. Scalapino. Rev. Mod. Phys. 84, 1383 (2012).

[16] D. Wulferding, M. Shay, G. Drachuck, R. Ofer, G. Bazalitsky, Z. Salman, P. Lemmens, A. Keren. Phys. Rev. B 90, 104511 (2014).

[17] A.S. Moskvin, Yu.D. Panov. J. Supercond. Nov. Magn. 32, 61 (2019).

Редактор Ю.Э. Китаев 